\title{
Positron scattering from pyridine
}

D. Stevens, T. J. Babij, J. R. Machacek, S. J. Buckman, M. J. Brunger, R. D. White, G. García, F. Blanco, L. Ellis-Gibbings, and J. P. Sullivan

Citation: The Journal of Chemical Physics 148, 144308 (2018); doi: 10.1063/1.5024246

View online: https://doi.org/10.1063/1.5024246

View Table of Contents: http://aip.scitation.org/toc/jcp/148/14

Published by the American Institute of Physics

\section{Articles you may be interested in}

Elastic scattering and vibrational excitation for electron impact on para-benzoquinone

The Journal of Chemical Physics 147, 244304 (2017); 10.1063/1.5010831

Electron-impact electronic-state excitation of para-benzoquinone

The Journal of Chemical Physics 148, 124312 (2018); 10.1063/1.5023494

Low-lying excited states by constrained DFT

The Journal of Chemical Physics 148, 144103 (2018); 10.1063/1.5018615

Investigations of the valence-shell excitations of molecular ethane by high-energy electron scattering

The Journal of Chemical Physics 148, 144313 (2018); 10.1063/1.5021695

Positron Scattering from Molecules: An Experimental Cross Section Compilation for Positron Transport Studies and Benchmarking Theory

Journal of Physical and Chemical Reference Data 46, 023102 (2017); 10.1063/1.4982827

Total cross section of furfural by electron impact: Experiment and theory

The Journal of Chemical Physics 147, 054301 (2017); 10.1063/1.4996462

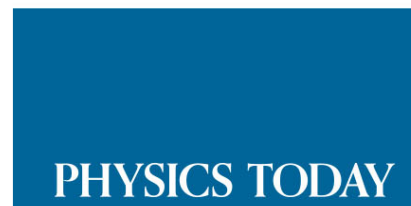




\title{
Positron scattering from pyridine
}

\author{
D. Stevens, ${ }^{1}$ T. J. Babij, ${ }^{1}$ J. R. Machacek, ${ }^{1, a)}$ S. J. Buckman, ${ }^{1}$ M. J. Brunger, ${ }^{2}$ R. D. White, ${ }^{3}$ \\ G. García, ${ }^{4}$ F. Blanco, ${ }^{5}$ L. Ellis-Gibbings, ${ }^{4}$ and J. P. Sullivan ${ }^{1}$ \\ ${ }^{1}$ Plasma Research Laboratory, Research School of Physics and Engineering, Australian National University, \\ Acton, Canberra 2601, Australia \\ ${ }^{2}$ College of Science and Engineering, Flinders University, Adelaide, SA, Australia \\ ${ }^{3}$ College of Science and Engineering, James Cook University, Townsville, Qld., Australia \\ ${ }^{4}$ Instituto de Física Fundamental, Consejo Superior de Investigaciones Científicas, Serrano 113-bis, \\ 28006 Madrid, Spain \\ ${ }^{5}$ Departamento de Física Atómica, Molecular y Nuclear, Universidad Complutense de Madrid, \\ E-28040 Madrid, Spain
}

(Received 30 January 2018; accepted 20 March 2018; published online 11 April 2018)

\begin{abstract}
We present a range of cross section measurements for the low-energy scattering of positrons from pyridine, for incident positron energies of less than $20 \mathrm{eV}$, as well as the independent atom model with the screening corrected additivity rule including interference effects calculation, of positron scattering from pyridine, with dipole rotational excitations accounted for using the Born approximation. Comparisons are made between the experimental measurements and theoretical calculations. For the positronium formation cross section, we also compare with results from a recent empirical model. In general, quite good agreement is seen between the calculations and measurements although some discrepancies remain which may require further investigation. It is hoped that the present study will stimulate development of $a b$ initio level theoretical methods to be applied to this important scattering system. Published by AIP Publishing. https://doi.org/10.1063/1.5024246
\end{abstract}

\section{INTRODUCTION}

Over the past decade and a half, the antimatter, and particularly the positron, has become a tool in the arsenal of oncologists for the detection and treatment of human cell disorders through the use of Positron Emission Tomography (PET) and positherapy. ${ }^{1}$ Hence, positron research into its interaction with biological systems and their constituents is important and significant in order to enhance our understanding and knowledge in this field. ${ }^{2,3}$ It is anticipated that such knowledge will ultimately contribute substantially to an improvement in the delivery of public health. Although research into the interaction between low-energy positrons and biological systems has been ongoing for over a decade, a complete understanding of these interactions and their effect on biological systems remains a developing field. ${ }^{4}$ On the other hand, substantially more is understood about the interaction ${ }^{5}$ and effect of lowenergy electrons, as reported by Sanche, ${ }^{6}$ for instance. While some aspects of charged particle interactions are common to both electrons and positrons, there are also significant differences and these will be discussed later. Of primary importance, in the context of these considerations, are the positron cross sections for scattering from biologically significant molecules and structures. At the heart of human genetics are the nucleobases, including thymine (DNA), cytosine (RNA and DNA), and uracil (RNA). These are each derived from the aromatic heterocyclic molecule pyrimidine which has already been the subject of a substantial experimental study of positron interactions in the work of Palihawadana et al. ${ }^{7}$ Pyridine differs

a)Electronic mail: Joshua.Machacek@anu.edu.au from pyrimidine insofar as it is the simplest azine, with a single $\mathrm{CH}$ group in the benzene ring being replaced by a nitrogen atom, whereas pyrimidine is a diazine, with two $\mathrm{CH}$ groups being replaced by nitrogen atoms at positions 1 and 3 of the ring.

Previous work within our group has included positron and/or electron cross-section measurements on pyrimidine, ${ }^{7-10}$ uracil, ${ }^{11}$ tetrahydrofuran (THF) ${ }^{12,13}$ formic acid, ${ }^{14}$ 3-hydroxy-tetrahydrofuran, ${ }^{15}$ and water. ${ }^{4,16,17}$ We have also recently studied how resilient biomolecules are to thermal decomposition, specifically for uracil and a range of PETrelated uracil derivatives, ${ }^{18}$ and found that even up to $200{ }^{\circ} \mathrm{C}$ their structural integrity remains. This is a crucial point as scattering studies such as ours require this, and it is also important for biomolecule collision experimentation in general. To the best of our knowledge, positron scattering experiments have not previously been undertaken with pyridine as the target molecule. ${ }^{19}$ The positron scattering measurements performed on pyridine in the present study are compared with results from calculations using the Independent Atom Model with the Screening Corrected Additivity Rule, including interference (IAM-SCAR+I) effects, together with a Born-type calculation to determine the contribution of rotational excitations. The main purpose of the present study is to extend the body of knowledge surrounding positron interactions with this family of molecules and thereby contribute to a more complete understanding of low-energy positron interactions with biological systems of potential relevance to PET scans. The inherent multicenter nature of the positron-molecule scattering problem has made $a b$ initio calculations largely intractable for molecules larger than hydrogen $\left(\mathrm{H}_{2}\right){ }^{20}$ As discussed above, 
a combination of techniques are currently being used to provide estimates of scattering cross sections for large molecules 5 but rely on phenomenological methods for channels such as positronium (Ps) formation. ${ }^{5}$ The lack of quantitative $a b$ initio level theoretical descriptions for positron scattering from molecules highlights the need for experimental investigations into positron scattering from a range of molecular targets, in order to explore the complicated multicenter nature of the scattering problem. One of the main aims of this investigation is, therefore, to provide a range of accurate and reliable scattering data, for a range of scattering processes, against which new theory might be benchmarked in order to test its accuracy. The structure of the remainder of this paper is as follows. In Sec. II, we briefly describe our apparatus and measurement techniques, while in Sec. III a description of our theoretical approach is provided. Thereafter, in Sec. IV, we present our results with a discussion of those results being found in Sec. V. Finally, conclusions are drawn in Sec. VI.

\section{EXPERIMENTAL}

The experimental apparatus has been described previously by Sullivan et $a .^{21}$ Briefly, a positron source of ${ }^{22} \mathrm{Na}$ with an activity of approximately $900 \mathrm{MBq}$ was used with the high energy positrons emanating from the source being moderated using a solid neon moderator. The moderated positron beam was magnetically guided into a 2-stage Surko trap where positrons were trapped and cooled via inelastic collisions with a buffer gas $\left(\mathrm{N}_{2}\right.$ and $\left.\mathrm{CF}_{4}\right)$. The output from the trap stage was a pulsed mono-energetic ( $80 \mathrm{meV}$ FWHM) beam at a repetition rate of $100 \mathrm{~Hz}$ which was directed towards the target/scattering section of the apparatus. The trap and target stages were contained in a uniform solenoidal magnetic field of approximately $530 \mathrm{G}$. The target stage consists of a retarding potential element, to reject positrons which have scattered with gases at the exit of the buffer gas trap, followed by a $5 \mathrm{~cm}$ gas cell which was connected to a reservoir of high-purity $(\geq 99.8 \%)$ liquid-phase pyridine molecules. Pyridine vapour was directed into the cell via Teflon tubing, and a manual needle valve was used to regulate the pressure of pyridine target gas within the scattering cell. Three freeze-pump-thaw cycles were used to remove any dissolved gas in the pyridine sample. Care was taken to ensure that the density of target molecules in the gas cell did not exceed that which would result in greater than $10 \%$ scatter inside the target cell, thereby limiting the occurrence of multiple scattering events. The analysis stage comprises a retarding potential analyser which is used to determine the parallel energy loss of the positron beam. This analyser stage is accommodated within a separate uniform solenoidal magnetic field, with a strength dictated by the need to separate elastic and inelastic collision events, as described in the work of Palihawadana et $a l^{7}$ In general, the scattering cross sections were determined using well-established techniques for scattering in a high magnetic field, as outlined by Sullivan et al. ${ }^{22}$ While these techniques allow the measurement of absolute cross section values, through the determination of the absolute target pressure, as discussed in detail elsewhere, ${ }^{23}$ the finite energy width of the incident magnetically guided beam sets limits on the angular range of the measurement.
TABLE I. Missing angle and percentage of (partial) cross section.

\begin{tabular}{lccc}
\hline $\begin{array}{l}\text { Energy } \\
(\mathrm{eV})\end{array}$ & $\begin{array}{c}\text { Minimum angle } \\
(\mathrm{deg})\end{array}$ & $\begin{array}{c}\text { Missing (\%) } \\
\text { IAM-SCAR+I }\end{array}$ & $\begin{array}{c}\text { Missing (\%) } \\
\text { IAM-SCAR+I+Born }\end{array}$ \\
\hline 1 & 17.5 & 8.1 & 44.1 \\
2 & 12.2 & 8.9 & 33.5 \\
3 & 10 & 7.8 & 25.9 \\
5 & 7.7 & 8.0 & 19.2 \\
10 & 5.4 & 5.9 & 14.8 \\
15 & 4.4 & 3.7 & 10.7 \\
20 & 3.8 & 2.7 & 7.7 \\
\hline \hline
\end{tabular}

Meaningful comparison between experimental and theoretical results must consider the angular range over which the experimental measurements are made, as discussed below and see Table I. The experimental data presented here are absolute, and their error bars account for both systematic and statistical uncertainties. Elastic scattering cross sections are effectively summed over some vibrational and rotational excitations, due to our finite energy resolution.

\section{CALCULATION PROCEDURE}

The screening corrected additivity rule, within the framework of the independent atom model (IAM-SCAR), as used in this study has been described in previous publications. ${ }^{4,24}$ It has been successfully applied in the past for several biologically relevant molecules, ${ }^{4,12,15,24}$ typically in the range of 0.1-10 $000 \mathrm{eV}$ incident energy. IAM-SCAR basically relies on the optical potential method, ${ }^{27,28}$ initially applied to the constituent atoms of the molecule, i.e., N, C, and H. The atomic scattering potential $^{29}$ is then represented by

$$
V(r)=V_{s}(r)+V_{p}(r)-i V_{a}(r) .
$$

The real part of Eq. (1) drives the elastic scattering dynamics and includes the electrostatic $\left(V_{s}(r)\right)$ and polarization $\left(V_{p}(r)\right)$ interactions. The imaginary part $\left(V_{a}(r)\right)$ describes all inelastic processes that are considered as absorptions from the incident positron beam. Owing to this last term in Eq. (1), the optical model potential method yields a complex phase shift $\delta_{l}=\lambda_{l}+i \mu_{l}$. This allows for the calculation of the atomic scattering amplitudes, from which the corresponding differential and integral elastic cross sections as well as the integral inelastic cross sections and therefore the total cross sections are derived. The static potential was obtained from the charge density derived from Hartree-Fock atomic wave functions, using a procedure analogous to that of Reid and Wadehra. ${ }^{30}$ The dipole plus quadrupole polarization potential was developed from that reported by McEachran et al. ${ }^{31}$ for Ne but scaled by constants in order to match the known dipole and quadrupole polarizabilities of the $\mathrm{C}, \mathrm{N}$, and $\mathrm{H}$ atoms (see Ref. 24 for details). The absorption potential accounts for the electronic excitations, positronium formation, and direct ionization. However, owing to the challenging nature of representing the Ps formation channel, the definition of the threshold energy for the absorption potential can be critical. Our recent improvements to the treatment of Ps formation are outlined in detail previously. ${ }^{4}$ In brief, we maintain the energy dependent threshold $\Delta(\mathrm{E})$, by 
necessity coinciding with the well-known Ps formation threshold of $\Delta_{p}=I-6.8 \mathrm{eV}$ (where I = ionization threshold) for lower energies and the lowest optically allowed excitation transition $\Delta$ for higher impact energies. The improvement is Eq. (2), detailing the smooth transition in threshold energy from low to high impact energy,

$$
\Delta(E)=\frac{\Delta-\left(\Delta-\Delta_{p}\right)}{\left[1+(E / 3 I-1)^{2}\right]} .
$$

Once we have calculated the atomic scattering amplitudes, ${ }^{31}$ the IAM-SCAR procedure ${ }^{15}$ gives the molecular scattering amplitudes, $F(\theta)$, from those of the constituent atoms, $f_{i}(\theta)$, according to the following expression:

$$
F(\theta)=\sum_{\text {atoms }} f_{i}(\theta) e^{i \mathbf{q} \cdot \mathbf{r}_{\mathbf{i}}},
$$

where the momentum transfer is $\mathbf{q}=\mathbf{k}_{\mathbf{f}}-\mathbf{k}_{\mathbf{i}}$ and the atomic positions are given by $\mathbf{r}_{\mathbf{i}}$. In this calculation, we incorporate the recent improvement of considering interference effects, as described in Ref. 32. This updated version is known as IAM-SCAR+I and basically provides the molecular differential cross section $\left(d \sigma_{\text {molecule }}^{\text {elastic }} / d \Omega\right)$ as a combination of the multicentre atomic amplitudes given by

$$
\begin{aligned}
\frac{d \sigma_{\text {molecule }}^{\text {elastic }}}{d \Omega}= & \sum_{i j} f_{i}(\theta) f_{j}^{*}(\theta) \frac{\sin q r_{i j}}{q r_{i j}}=\sum_{i}\left|f_{i}(\theta)\right|^{2} \\
& +\sum_{i \neq j} f_{i}(\theta) f_{j}^{*}(\theta) \frac{\sin q r_{i j}}{q r_{i j}} .
\end{aligned}
$$

Here the interference term is the second summation in Eq. (4). In this case, $q \equiv|\mathbf{q}|=2 k \sin \theta / 2$ is the momentum transfer and $r_{i j}$ is the distance between atoms $i$ and $j$. By integrating Eq. (4), the corresponding molecular integral cross sections are represented by

$$
\sigma_{\text {molecule }}^{\text {total }}=\sum_{\text {atoms }} s_{i} \sigma_{\text {atomi }}^{\text {total }}+\sigma^{\text {interference }} .
$$

The factor $s_{i}$ is a screening correction, reducing the contribution of each atom to the total molecular cross section $\left(0 \leq s_{i} \leq 1\right)$ based on the position of the atom within the molecule. This accounts for the fact that as the energy of the incoming particle decreases, the atomic cross sections overlap requiring a reduction of their relative contribution to the summation (see Ref. 32 for details). The remaining term in Eq. (5) is determined from integration of the second summation in Eq. (4),

$$
\sigma_{\text {interference }} \equiv \int d \Omega \sum_{i \neq j} f_{i}(\theta) f_{j}^{*}(\theta) \frac{\sin q r_{i j}}{q r_{i j}} .
$$

As shown in Ref. 30, including interference terms in the calculation of both integral and differential cross sections for molecular targets eliminates the inconsistency between the differential and integral cross section values, which is inherent to the IAM-SCAR method. This means that no additional normalisation procedure is required by the IAMSCAR+I approach in order to fulfil the optical theorem. ${ }^{32}$ The IAM-SCAR+I procedure does not consider nuclear motions in the molecule, and therefore neither vibrational nor rotational excitations are included in the calculation. Vibrational cross sections in a target such as pyridine are expected to only make a small contribution to scattering over the energy range considered in this work. As pyridine possesses a permanent dipole moment, rotational excitations may be induced by the colliding positrons at any incident energy and constitute an important inelastic channel. ${ }^{25,26}$ For this reason, an independent rotational excitation calculation, within the framework of the Born approximation with Dickinson corrections for the larger scattering angles (see Ref. 33 for details), has been carried out. In these conditions, we assume that the initial rotational excited state population of the molecular target follows the temperature dependent Boltzmann distribution and an increment of \pm 1 in the rotational quantum number is induced by each positron collision. We are therefore considering average transitions between the initial and final rotational state distributions, for which the effective energy transferred, at a given temperature, depends on the rotational constants (see Ref. 34). In the case of pyridine, we obtained an effective rotational excitation energy of $1.09 \mathrm{meV}$ at room temperature $(300 \mathrm{~K})$. The rotational excitation cross sections calculated with the above procedure can be incoherently added to the IAM-SCAR+I cross section to obtain the grand total cross section, including rotations, denoted by IAM-SCAR+I+Born.

\section{RESULTS}

Pyridine $\left(\mathrm{C}_{5} \mathrm{H}_{5} \mathrm{~N}\right)$ has an ionization potential (IP) of $9.26 \mathrm{eV}^{35}$ and a positronium formation threshold at an energy of $2.46 \mathrm{eV}$ which is $6.8 \mathrm{eV}$ (the binding energy of positronium) below the IP. Measurements for this study have been made at impact energies below $20 \mathrm{eV}$ impact energy and include grand total cross sections, total elastic cross sections (TECS), total inelastic cross sections (TICS), and positronium (Ps) formation cross sections.

\section{A. Grand total cross section}

The grand total cross section data for positron scattering from pyridine below $20 \mathrm{eV}$ impact energy are shown in Fig. 1 and listed in Table II. Here we compare our experimental measurements with results from our IAM-SCAR+I calculations. Calculations including the Born approximation to determine rotational excitations (IAM-SCAR+I+Born) are also included in this figure. For experiments such as this conducted in a high magnetic field, the finite energy width of the positron beam limits the angular range of the measurements, ${ }^{23}$ which consequently affects the measurement of the total (integral elastic or integral inelastic) and grand total cross sections, as shown in Table I. To allow for this, we estimate the forward scattered portion of the elastic and rotational contributions (we cannot distinguish between these with the present energy resolution) which are not measured. At low energies, where the missing angular range is the largest, the experiment misses a significant portion of the "true" total cross section. This decreases in significance as the impact energy increases, as the missing angle is correspondingly reduced. From the calculation, most of this correction is due to the contribution of rotational scattering to the total cross section. 


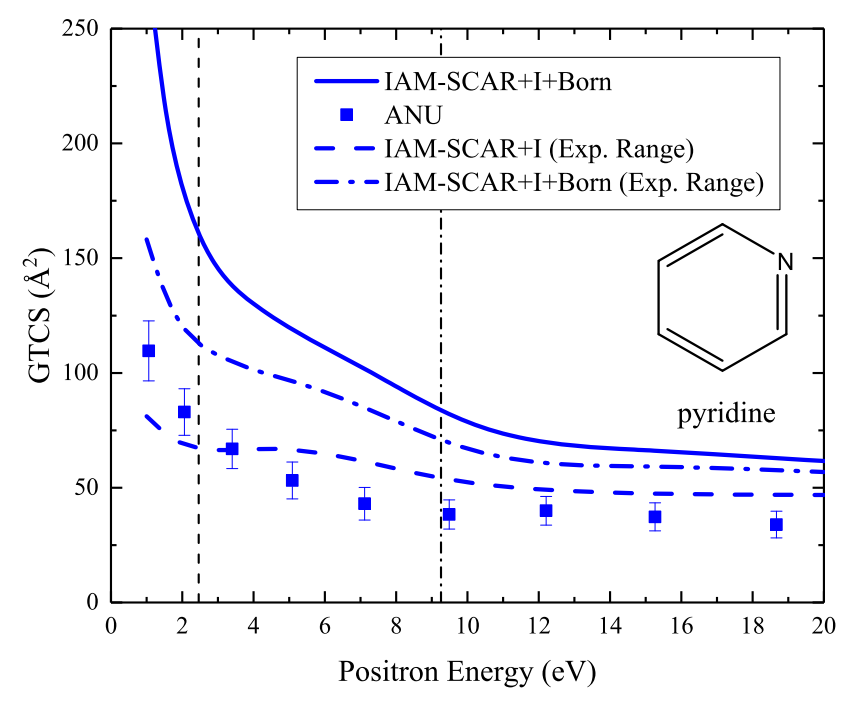

FIG. 1. Results of the grand total cross section for positron scattering from pyridine. Comparison of the IAM-SCAR+I+Born calculations (solid line), the measured total scattering cross section for positron scattering from pyridine (solid squares), and the IAM-SCAR+I calculation (dashed blue line) and the IAM-SCAR+I+Born calculation (dotted-dashed line), both of which have been modified to represent the equivalent angular range of the experimental measurements. The vertical dashed line indicates the positronium formation threshold, and the vertical dotted-dashed line indicates the ionization potential. Tabular data are available in Table II.

It can be seen from Fig. 1 that the agreement between experiment and the IAM-SCAR+I calculation is better than with the IAM-SCAR+I+Born calculation, over the experimental energy and angular ranges considered. This represents a query as to the validity of the Born approximation for accurate calculation of the contributions of rotational cross sections. Note that this is an important point as the disagreement implies that the rotational contributions are overestimated by the Born approximation, which is clear despite the limited contribution to the experimental data from scattering in the forward direction. Recent modeling of transport in THF has shown the Born approximation to overestimate the rotational contributions, ${ }^{36}$ which is consistent with the present results.

\section{B. Total elastic and total inelastic cross sections}

The present experimental and theoretical (IAM-SCAR+I) results of the total elastic (TECS) and the theoretical (IAM-SCAR+I) total inelastic (TICS) positron scattering cross sections are shown in Fig. 2 and listed in Table III, and the TICS values represent the sum of the electronic excitations, positronium formation, and ionization channels. Under these conditions, at low energy, there is little contribution to the scattering process from inelastic collisions, but as the energy increases to $20 \mathrm{eV}$, they contribute strongly and comprise almost half the total cross section magnitude at this energy. In contrast to the measurement of the elastic channel, the experimental technique captures the inelastic channel without loss since the excitation energy is much greater than the energy width of the incident positron beam. The solid red line in the figure is the (reduced) theoretical cross section when it is integrated over the appropriate experimental angular range, as described above. In general, the agreement between the two below the positronium formation threshold is good. However, the two results diverge at the Ps formation threshold, before appearing to converge again as the energy approaches the ionization potential. This can be understood as a limitation of the IAM-SCAR+I procedure which uses the positronium formation thresholds of the constituent atoms instead of that of the molecule. Note that this threshold is lower for pyridine $(2.46 \mathrm{eV})$ than for those corresponding to the constituent $\mathrm{C}, \mathrm{N}$, and $\mathrm{H}$ atoms. It is well known (see Ref. 33 and references therein) that, for a given incident energy, not

TABLE II. Present grand total cross section results of positron scattering from pyridine, in units of $10^{-16} \mathrm{~cm}^{2}$, and the uncertainties represent the absolute error on the measured data; see Fig. 1.

\begin{tabular}{|c|c|c|c|c|c|}
\hline $\begin{array}{l}\text { Energy } \\
(\mathrm{eV})\end{array}$ & IAM-SCAR+I+Born & $\begin{array}{c}\text { IAM-SCAR+I } \\
\text { (expt. range) }\end{array}$ & $\begin{array}{c}\text { IAM-SCAR+I+Born } \\
\text { (expt. range) }\end{array}$ & $\begin{array}{l}\text { GTCS } \\
\text { (expt.) }\end{array}$ & Uncert. \\
\hline 1 & 282.8 & 158.1 & 81.1 & & \\
\hline 1.1 & & & & 109.6 & 13.1 \\
\hline 1.5 & 217.8 & 135.3 & 73.7 & & \\
\hline 2 & 181.2 & 120.5 & 68.4 & & \\
\hline 2.1 & & & & 83.0 & 10.2 \\
\hline 3 & 145.6 & 107.8 & 65.8 & & \\
\hline 3.4 & & & & 66.9 & 8.6 \\
\hline 4 & 130.2 & 101.4 & 66.8 & & \\
\hline 5 & 119.8 & 96.8 & 67.2 & & \\
\hline 5.1 & & & & 53.2 & 8.0 \\
\hline 7 & 102.8 & 85.6 & 62.6 & & \\
\hline 7.1 & & & & 43.0 & 7.1 \\
\hline 9.5 & & & & 38.4 & 6.4 \\
\hline 10 & 78.7 & 67.0 & 50.1 & & \\
\hline 12.2 & & & & 40.0 & 6.2 \\
\hline 15 & 66.4 & 59.3 & 47.1 & & \\
\hline 15.3 & & & & 37.3 & 6.1 \\
\hline 18.7 & & & & 33.9 & 5.8 \\
\hline 20 & 61.6 & 56.9 & 46.8 & & \\
\hline
\end{tabular}




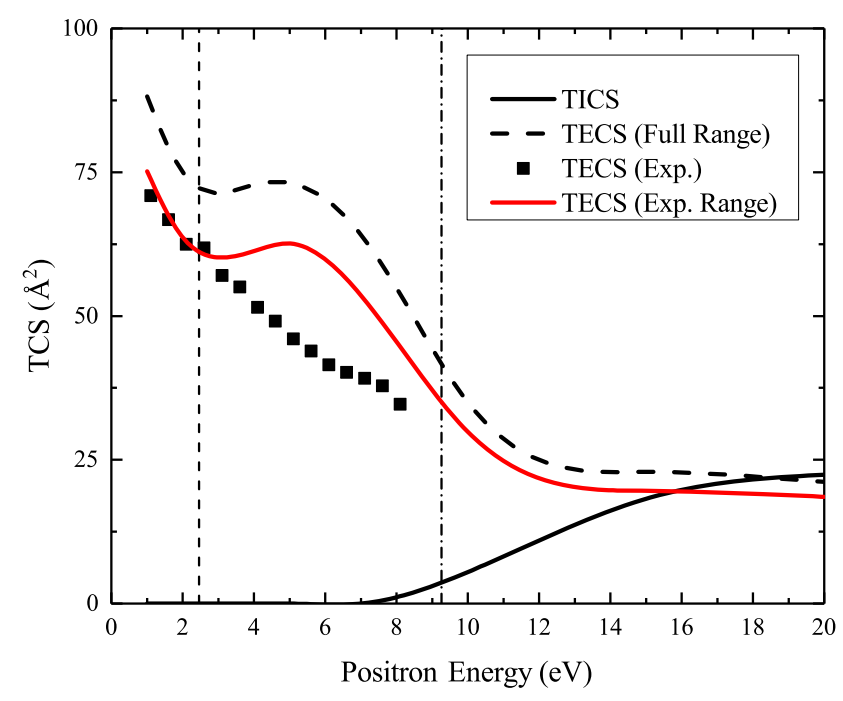

FIG. 2. Total elastic (TECS) and total inelastic (TICS) scattering cross sections for positron scattering from pyridine $(\mathrm{C} 5 \mathrm{H} 5 \mathrm{~N})$ : solid squares present measurements of TECS; dashed line presents IAM-SCAR+I calculation of TECS (0-180 deg); solid red line presents IAM-SCAR+I calculation over the experimental angular range; and solid black line presents IAM-SCAR+I calculation of the TICS. The vertical dashed line indicates the positronium formation threshold, and the vertical dotted-dashed line indicates the ionization potential. See the text for discussion of the discrepancy in the measured and calculated TECS. Tabular data are available in Table III.

including appropriately for the open inelastic channels at that energy leads to an overestimation of the elastic cross section. Once positronium formation is included in the calculation,

TABLE III. Present TECS and TICS results of positron scattering from pyridine, in units of $10^{-16} \mathrm{~cm}^{2}$, and the uncertainties represent the absolute error on the measured data; see Fig. 2.

\begin{tabular}{|c|c|c|c|c|c|}
\hline $\begin{array}{l}\text { Energy } \\
(\mathrm{eV})\end{array}$ & $\begin{array}{l}\text { Elastic } \\
\text { (theory) }\end{array}$ & $\begin{array}{l}\text { Reduced angular } \\
\text { range }\end{array}$ & $\begin{array}{c}\text { TICS } \\
\text { (theory) }\end{array}$ & $\begin{array}{l}\text { TECS } \\
\text { (expt.) }\end{array}$ & Uncert \\
\hline 1 & 88.2 & 75.1 & 0.0 & & \\
\hline 1.1 & & & & 70.9 & 2.1 \\
\hline 1.5 & 80.6 & 68.6 & 0.0 & & \\
\hline 1.6 & & & & 66.8 & 2.1 \\
\hline 2 & 75.0 & 63.7 & 0.0 & & \\
\hline 2.1 & & & & 62.5 & 2.0 \\
\hline 2.6 & & & & 61.8 & 2.0 \\
\hline 3 & 71.4 & 60.2 & 0.0 & & \\
\hline 3.1 & & & & 57.1 & 1.9 \\
\hline 3.6 & & & & 55.0 & 1.9 \\
\hline 4 & 72.8 & 61.3 & 0.0 & & \\
\hline 4.1 & & & & 51.5 & 1.9 \\
\hline 4.6 & & & & 49.1 & 1.8 \\
\hline 5 & 73.1 & 62.6 & 0.0 & & \\
\hline 5.1 & & & & 46.0 & 1.8 \\
\hline 5.6 & & & & 43.9 & 1.8 \\
\hline 6.1 & & & & 41.5 & 1.8 \\
\hline 6.6 & & & & 40.2 & 1.8 \\
\hline 7 & 64.1 & 53.6 & 0.0 & & \\
\hline 7.1 & & & & 39.2 & 1.8 \\
\hline 7.6 & & & & 37.9 & 1.7 \\
\hline 8.1 & & & & 34.7 & 1.7 \\
\hline 10 & 35.0 & 29.9 & 5.5 & & \\
\hline 15 & 22.9 & 19.6 & 18.2 & & \\
\hline 20 & 21.2 & 18.6 & 22.4 & & \\
\hline
\end{tabular}

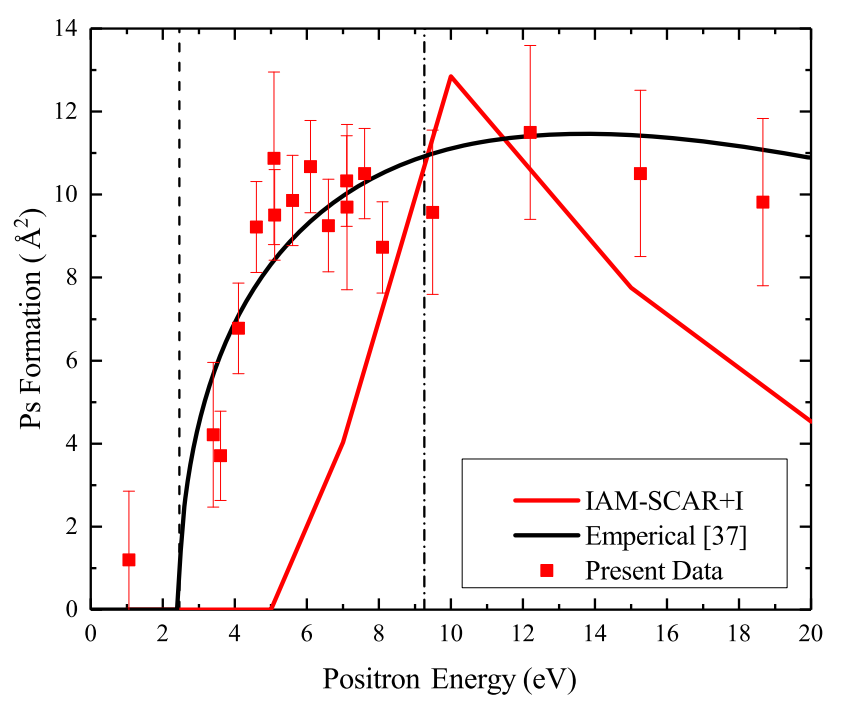

FIG. 3. Positronium (Ps) formation cross section for positron scattering from pyridine $\left(\mathrm{C}_{5} \mathrm{H}_{5} \mathrm{~N}\right)$ : red squares present results; black line presents the parametric model (dipole polarisability) of Ps formation; ${ }^{37}$ red line presents IAM-SCAR+I results. Tabular data are available in Table IV.

above $7 \mathrm{eV}$, the experimental and theoretical data tend to converge for increasing energies.

\section{Positronium (Ps) formation}

The results of the positronium formation cross section are presented in Fig. 3 and listed in Table IV. The experimental results are compared to the present phenomenological IAM-SCAR+I result and an empirical scaling model ${ }^{37}$

TABLE IV. Present positronium formation cross sections of positron scattering from pyridine, in units of $10^{-16} \mathrm{~cm}^{2}$, and the uncertainties are the absolute error on the measured data; see Fig. 3.

\begin{tabular}{lcccc}
\hline \hline Energy $(\mathrm{eV})$ & Theory & Empirical & Exp. Ps formation & Uncert. \\
\hline 1 & 0 & 0 & & \\
1.1 & 0 & 0 & 1.2 & 1.7 \\
1.5 & 0 & 0 & & \\
2 & 0 & 0 & & \\
2.5 & 0 & 1.4 & & \\
3.4 & 0 & 5.7 & 4.2 & 1.7 \\
3.6 & 0 & 6.1 & 3.7 & 1.1 \\
4.1 & 0 & 7.1 & 6.8 & 1.1 \\
4.6 & 0 & 7.8 & 9.2 & 1.1 \\
5.1 & 0 & 8.4 & 9.8 & 1.0 \\
5.6 & 0 & 8.9 & 9.9 & 1.1 \\
6.1 & 0 & 9.4 & 10.7 & 1.1 \\
6.6 & 0 & 9.7 & 9.3 & 1.1 \\
7 & 4.0 & 10.0 & & \\
7.1 & & 10.0 & 10.2 & 1.0 \\
7.6 & & 10.3 & 10.5 & 1.1 \\
8.1 & & 10.5 & 8.7 & 1.1 \\
9.5 & & 11.0 & 9.6 & 2.0 \\
10 & 12.9 & 11.1 & & \\
12.2 & & 11.4 & 11.5 & 2.1 \\
15 & 7.8 & 11.4 & & \\
15.3 & & 11.4 & 10.5 & 2.0 \\
18.7 & & 11.1 & 9.8 & 2.0 \\
20 & 4.5 & 10.9 & & \\
\hline \hline
\end{tabular}


based on the dipole polarizability, which for pyridine is $64.06 a_{\circ}^{3}, 35$ using the suggested shape parameters. ${ }^{37}$ The general trend from experimental results for the Ps formation cross section, for both atoms and molecules, is a rapid turn-on of the cross section with a peak in the cross section at approximately $10 \mathrm{eV}$ above the threshold. This is seen to hold in the case of the present measurements for pyridine. In this case, we see very good agreement with the recent scaling model, ${ }^{37}$ but only marginal agreement with the phenomenological model used in the IAM-SCAR+I calculation. The IAM-SCAR+I calculation does not closely reproduce the energy dependence but does provide a reasonable estimate of the peak value of the Ps formation cross section and to some extent the peaked nature of the cross section as a function of energy. This situation can again be explained, at least in part, by the fact that we use the atomic Ps formation thresholds instead of the molecular value. This also highlights that in both the total elastic and Ps formation cross section cases, the largest discrepancy between the experiment and theory is between the Ps formation threshold and the (direct) ionization threshold.

\section{DISCUSSION}

The level of agreement between the experimentally measured values and the IAM-SCAR+I calculations for positron scattering cross sections from pyridine, as presented in this paper, is generally quite good. There remain, however, some discrepancies between the two sets of data. The largest discrepancy is observed between the energy dependence of the measured and IAM-SCAR+I Ps formation cross sections. This is perhaps not surprising, given the nature of the calculation. By contrast, a different empirical model of the Ps formation cross section, based on the molecular dipole polarisability, is in very good agreement with regard to both the energy dependence and magnitude. Clearly more work is needed to understand the Ps formation process in positron scattering, in particular, in the energy region between the Ps formation and the direct ionisation thresholds. While it appears that the cross section is very well modeled empirically, with a dependence only on the dipole polarisability, more theoretical work is warranted to better understand this from a fundamental point of view. It should be noted, however, that at the grand total cross section level, the energy dependence of IAM-SCAR+I and experimental measurement results are in good agreement, so this discrepancy is not readily apparent at that level of comparison. There is also disagreement between the theory and experiment for total elastic scattering in the region between the positronium formation and ionisation thresholds. This is likely due to the representation of positronium formation in the calculation and therefore warrants further consideration for future work. While the theory presented here does not consider vibrational excitations of pyridine, at least some of the normal modes are included in the experiment, in the sense that they are incorporated into the measurement of elastic scattering. As a result, the agreement we observe, in magnitude and shape, between the experiment and theory below the Ps formation threshold $(\approx 3 \mathrm{eV})$ implies that vibrational excitation in this energy region is relatively small compared to the elastic channel.

\section{CONCLUSIONS}

This paper presents the first set of cross section measurements for positron scattering from pyridine. Additionally, theoretical calculations using the IAM-SCAR+I and IAM-SCAR+I+Born methods were presented. Generally quite good agreement was observed between the experimental data and the theoretical calculations across the energy range investigated. However, discrepancies were observed at energies between the positronium formation and the direct ionisation thresholds which were not observed using a new empirical model of positronium formation. Comparison at low energies suggests that vibrational excitation plays only a small role in the scattering process in the energy region investigated. Further measurements of positron scattering with other heterocyclic organic molecules may elucidate some of the discrepancies between measurement and theory, and we recommend they be undertaken. Of particular importance is a more complete description of the positronium formation cross section in molecules. Finally, the reported data further build the database $^{19}$ of positron cross-section information for biologically relevant molecules. This is a critical aspect of this study in order to encourage the development of ab initio level positronmolecule scattering computations, by providing theorists with a range of accurate and reliable measurements against which they can benchmark their results.

\section{ACKNOWLEDGMENTS}

The authors would like to acknowledge the Australian Research Council (ARC) Discovery Programmes for funding support (No. DP140102854) and Ross Tranter for technical support for the experimental apparatus. L.E.G., F.B., and G.G. also acknowledge partial financial support from the Spanish Ministry MINECO (No. FIS201680440) and the European Union ITN-Marie Curie programme (No. ARGENT-608163).

${ }^{1}$ R. M. Moadel, R. H. Weldon, E. B. Katz, P. Lu, J. Mani, M. Stahl, M. D. Blaufox, R. G. Pestell, M. J. Charron, and E. Dadachova, Cancer Res. 65, 698 (2005), ISSN: 0008-5472.

${ }^{2}$ R. D. White, W. Tattersall, G. Boyle, R. E. Robson, S. Dujko, Z. L. Petrovic, A. Bankovic, M. J. Brunger, J. P. Sullivan, S. J. Buckman, and G. García, Appl. Radiat. Isot. 83, 77 (2014).

${ }^{3}$ Z. Lj. Petrović, S. Marjanović, S. Dujko, A. Banković, G. Malović, S. Buckman, G. García, R. White, and M. Brunger, Appl. Radiat. Isot. 83, 148 (2014).

${ }^{4}$ F. Blanco, A. Roldan, K. Krupa, R. P. McEachran, R. D. White, S. Marjanović, Z. L. Petrović, M. J. Brunger, J. R. Machacek, S. J. Buckman, J. P. Sullivan, L. Chiari, P. Limão-Vieira, and G. García, J. Phys. B 49, 145001 (2016).

${ }^{5}$ M. J. Brunger, Int. Rev. Phys. Chem. 36, 333 (2017).

${ }^{6}$ L. Sanche, Eur. Phys. J. D 35, 367 (2005).

${ }^{7}$ P. Palihawadana, R. Boadle, L. Chiari, E. K. Anderson, J. R. Machacek, M. J. Brunger, S. J. Buckman, and J. P. Sullivan, Phys. Rev. A 88, 012717 (2013).

${ }^{8}$ Z. Mašín, J. D. Gorfinkiel, D. B. Jones, S. M. Bellm, and M. J. Brunger, J. Chem. Phys. 136, 144310 (2012).

${ }^{9}$ D. B. Jones, S. M. Bellm, P. Limão-Vieira, and M. J. Brunger, Chem. Phys. Lett. 535, 30 (2012).

${ }^{10}$ M. C. Fuss, A. G. Sanz, F. Blanco, J. C. Oller, P. Limão-Vieira, M. J. Brunger, and G. García, Phys. Rev. A 88, 042702 (2013).

${ }^{11}$ E. K. Anderson, R. A. Boadle, J. R. Machacek, L. Chiari, C. Makochekanwa, S. J. Buckman, M. J. Brunger, G. Garcia, F. Blanco, and O. Ingolfsson, J. Chem. Phys. 141, 034306 (2014). 
${ }^{12}$ L. Chiari, E. Anderson, W. Tattersall, J. R. Machacek, P. Palihawadana, C. Makochekanwa, J. P. Sullivan, G. García, F. Blanco, R. P. McEachran, M. J. Brunger, and S. J. Buckman, J. Chem. Phys. 138, 074301 (2013).

${ }^{13}$ T. P. T. Do, M. Leung, M. Fuss, G. Garcia, F. Blanco, K. Ratnavelu, and M. J. Brunger, J. Chem. Phys. 134, 144302 (2011).

${ }^{14}$ C. Makochekanwa, A. Bankovic, W. Tattersall, A. Jones, P. Caradonna, D. S. Slaughter, M. J. Brunger, Z. Petrovic, J. P. Sullivan, and S. J. Buckman, New J. Phys. 11, 103036 (2009).

${ }^{15}$ L. Chiari, P. Palihawadana, J. R. Machacek, C. Makochekanwa, G. García, F. Blanco, R. P. McEachran, M. J. Brunger, S. J. Buckman, and J. P. Sullivan, J. Chem. Phys. 138, 074302 (2013).

${ }^{16}$ W. Tattersall, L. Chiari, J. R. Machacek, E. Anderson, R. D. White, M. J. Brunger, S. J. Buckman, G. Garcia, F. Blanco, and J. P. Sullivan, J. Chem. Phys. 140, 044320 (2014).

${ }^{17}$ W. J. Tattersall, D. G. Cocks, G. J. Boyle, M. J. Brunger, S. J. Buckman, G. García, Z. L. Petrović, J. P. Sullivan, and R. D. White, Plasma Sources Sci. Technol. 26, 45010 (2017).

${ }^{18}$ T. M. Maddern, V. Jamier, J. R. Brunton, M. J. Brunger, C. Papamicaël, S. V. Smith, and S. J. Buckman, Int. J. Mass Spectrom. 409, 73 (2016).

${ }^{19}$ M. J. Brunger, S. J. Buckman, and K. Ratnavelu, J. Phys. Chem. Ref. Data 46, 023102 (2017).

${ }^{20}$ M. C. Zammit, D. V. Fursa, J. S. Savage, I. Bray, L. Chiari, A. Zecca, and M. J. Brunger, Phys. Rev. A 95, 022707 (2013).

${ }^{21}$ J. P. Sullivan, A. Jones, P. Caradonna, C. Makochekanwa, and S. J. Buckman, Rev. Sci. Instrum. 79, 113105 (2008).

${ }^{22}$ J. P. Sullivan, S. J. Gilbert, J. P. Marler, R. G. Greaves, S. J. Buckman, and C. M. Surko, Phys. Rev. A 66, 042708 (2002).

${ }^{23}$ J. P. Sullivan, C. Makochekanwa, A. Jones, P. Caradonna, D. S. Slaughter, J. Machacek, R. P. McEachran, D. W. Mueller, and S. J. Buckman, J. Phys. B: At., Mol. Opt. Phys. 44, 035201 (2011).
${ }^{24}$ L. Chiari, A. Zecca, S. Girardi, E. Trainotti, G. García, F. Blanco, R. P. McEachran, and M. J. Brunger, J. Phys. B: At., Mol. Opt. Phys. 45, 215206 (2012).

${ }^{25}$ A. G. Sanz, M. C. Fuss, F. Blanco, Z. Mašín, J. D. Gorfinkiel, R. P. McEachran, M. J. Brunger, and G. García, Phys. Rev. A 88, 062704 (2013).

${ }^{26}$ L. Chiari, A. Zecca, F. Blanco, G. García, and M. J. Brunger, Phys. Rev. A 91, 012711 (2015).

${ }^{27}$ F. Blanco and G. García, Phys. Rev. A 67, 022701 (2003).

${ }^{28}$ F. Blanco and G. García, Phys. Lett. A 317, 458 (2003).

${ }^{29}$ R. P. McEachran, J. P. Sullivan, S. J. Buckman, M. J. Brunger, M. C. Fuss, A. Muñoz, F. Blanco, R. D. White, Z. L. Petrović, P. Limào-Vieira, and G. García, J. Phys. B: At., Mol. Opt. Phys. 45, 045207 (2012).

${ }^{30}$ D. D. Reid and J. M. Wadehra, J. Phys. B: At., Mol. Opt. Phys. 29, L127 (1996).

${ }^{31}$ R. P. McEachran, D. L. Morgan, A. G. Ryman, and A. D. Stauffer, J. Phys. B: At., Mol. Opt. Phys. 10, 663 (1977).

${ }^{32}$ F. Blanco, L. Ellis-Gibbings, and G. Garcia, Chem. Phys. Lett. 645, 71 (2016).

${ }^{33}$ A. G. Sanz, M. C. Fuss, F. Blanco, F. Sebastianelli, F. A. Gianturco, and G. García, J. Chem. Phys. 137, 124103 (2012).

${ }^{34}$ A. Sieradzka, F. Blanco, M. C. Fuss, Z. Masin, J. Gorfinkiel, and G. Garcia, J. Phys. Chem. A 118, 6657 (2014).

${ }^{35}$ NIST Standard Reference Database Number 101, Release 18, edited by R. D. Johnson III (NIST Computational Chemistry Comparison and Benchmark Database, 2016), http://cccbdb.nist.gov/.

${ }^{36}$ M. J. E. Casey, J. de Urquijo, L. N. S. Loli, D. G. Cocks, G. J. Boyle, D. B. Jones, M. J. Brunger, and R. D. White, J. Chem. Phys. 147, 195103 (2017).

${ }^{37}$ J. R. Machacek, F. Blanco, G. Garcia, S. J. Buckman, and J. P. Sullivan, J. Phys. B: At., Mol. Opt. Phys. 49, 064003 (2016). 